\title{
Full Model-Free Control Architecture for Hybrid UAVs
}

\author{
Jacson M. O. Barth ${ }^{1}$, Jean-Philippe Condomines ${ }^{1}$, \\ Jean-Marc Moschetta ${ }^{2}$, Aurélien Cabarbaye ${ }^{1}$, Cédric Join ${ }^{3,5}$ and Michel Fliess ${ }^{4,5}$
}

\begin{abstract}
This paper discusses the development of a control architecture for hybrid Unmanned Aerial Vehicles (UAVs) based on model-free control (MFC) algorithms. Hybrid UAVs combine the beneficial features of fixed-wing UAVs with Vertical TakeOff and Landing (VTOL) capabilities to perform five different flight phases during typical missions, such as vertical takeoff, transitioning flight, forward flight, hovering and vertical landing. Based on model-free control principles, a novel control architecture that handles the hybrid UAV dynamics at any flight phase is presented. This unified controller allows autonomous flights without discontinuities of switching for the entire flight envelope with position tracking, velocity control and attitude stabilization. Simulation results show that the proposed control architecture provides an effective control performance for the entire flight envelope and excellent disturbance rejections during the critical flight phases, such as transitioning and hovering flights in windy conditions.
\end{abstract}

\section{INTRODUCTION}

Autonomous flight of Unmanned Aerial Vehicles (UAVs) remains an interesting and active research domain after decades of studies in the subject. The wide variety of missions involving UAVs, combined with advances in the fields of materials and computer science, have contributed to the design of new UAV configurations. Therefore, the drawbacks of rotatory-wing UAVs (e.g. helicopters, quadrotors and multi-rotors) in terms of endurance and range, with the lack of capability to take-off and landing from small areas of fixed-wing UAVs have also encouraged the development of a new UAV class namely hybrid UAV. This hybrid UAV configuration is able to perform complex flight missions in windy environments through its large flight envelope, as described in Fig. 1, with vertical take-off and landing with fast and efficient forward flight to reach a distant position. Although the combination of two different UAV configurations in a single one provides a wider application field, the control system needs to consider the particularities of each one in order to properly carry out the position tracking, velocity control and attitude stabilization during the entire flight envelope for a given mission.

\footnotetext{
${ }^{1}$ Are with ENAC, Université de Toulouse, France. [jacsonmiguel.olszanecki-barth; jean-philippe.condomines]@enac.fr

${ }^{2}$ Is with the Department of Aerodynamics, Energetics and Propulsion, Institut Supérieur de l'Aéronautique et de l'Espace, 31400 Toulouse, France. jean-marc.moschetta@isae-supaero.fr

${ }^{3}$ Is with the CRAN (CNRS, UMR 7039)), Université de Lorraine, BP 239, 54506 Vandœuvre-lès-Nancy, France. cedric.join@univ-lorraine.fr

${ }^{4}$ Is with the LIX (CNRS, UMR 7161), École polytechnique, 91128 Palaiseau, France. Michel.Fliess@polytechnique.edu

${ }^{5}$ Are with the AL.I.E.N (ALgèbre pour Identification \& Estimation Numérique), 7 rue Maurice Barrès, 54330 Vézelise, France. [michel.fliess; cedric.join]@alien-sas.com
}

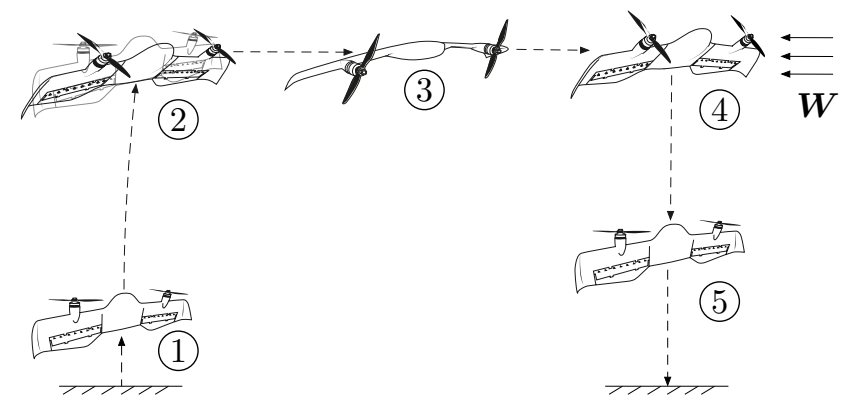

Fig. 1: Typical flight modes of Hybrid Unmanned Aerial Vehicles: 1 - Vertical take-off; 2 - Transition; 3 - Forward flight; 4 - Hover flight; 5 - Vertical landing. The vector $\boldsymbol{W}$ represents the wind disturbances.

While attitude stabilization for hovering and forward flights are commonly researched and quite known [1] [2], the attitude stabilization during the transition phase poses a major challenge due to the fast variations of aerodynamic forces and moments at high angles of attack. Sometimes considered as an undesirable and temporally transient between hover and forward flight [3], the transition phase needs to be continuously stabilized in order to ensure a smooth and safe flight. One of the control approach to handle the attitude stabilization relies on adaptive control laws, [4] proposes an adaptive quaternion algorithm in order to avoid singularities during the control of the entire flight envelope. On the other hand, instability problems with adaptive control methods can still exist with regard to unmodeled dynamics or inaccurate models in the adaptation law of the controller's parameters. Conventional model-based controls are also designed, for instance, nonlinear feedback techniques [5] [6], based on Lyapunov designs [7], linear optimal controls [8], linear optimal control with gain scheduling techniques [9] which require sophisticated wind-tunnel characterizations to get an understanding of forces and moments acting on the system. The performance of model-based controllers differs primarily in the fidelity with which the plant is modeled. Accurate modeling of aerodynamic forces and moments of a partially stalled wing is a difficult and time consuming task. Additionally, these models usually require several flight measurements that are hard to obtain such as angle of attack during hovering flights, and low airspeed due to small dynamic pressures. In terms of flight validation, [10] proposes a simple control strategy based on Proportional Integral and Derivative (PID) gains in order to control the 


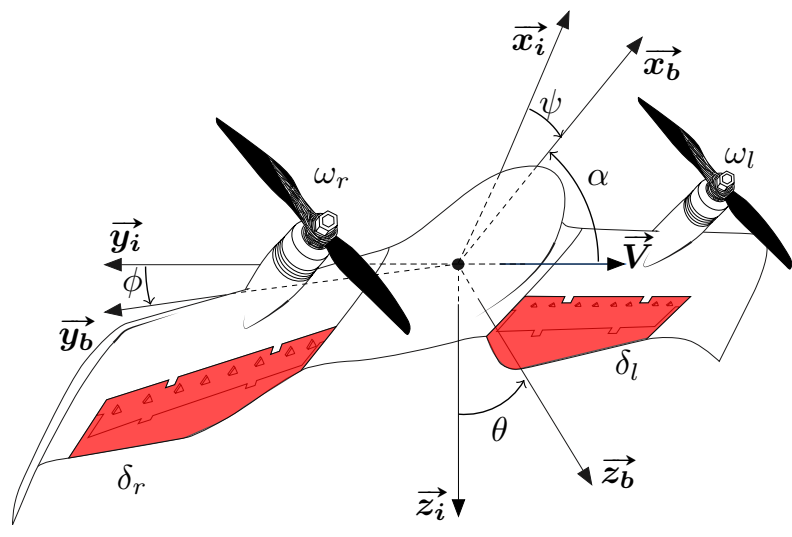

Fig. 2: Illustration of the used coordinate frames, angles and actuators. The Darko hybrid UAV was designed by Murat Bronz at ENAC.

position and the attitude of a hybrid UAV. Although simple to tune without the knowledge of the model, PID controllers are limited in terms of disturbance rejection. Another flight experiment, employing a cascaded control architecture composed by Incremental Nonlinear Dynamic Inversion (INDI) algorithms, was developed and flight-tested to demonstrate the performance of a such controller for position tracking, velocity control and attitude stabilization [11]. This sensorbased control approach needs a prior identification of the actuator effectiveness which are computed from real flight data implying a prior adjustment of the controller's gains. As an alternative, some control approaches can handle the system dynamics without requiring any model, such as the Model-Free Control (MFC) approach. Apart from aerospace applications [12] [13], including our previous work [14] [15], MFC was also applied on a wide list of different industrial cases sometimes providing patents, see [16] and its references for additional information. However to the best of our knowledge, this control methodology was never designed for hybrid UAVs. Thus, the new contributions presented in this paper, with respect to our previous work, are :

- guidance formulation (position and velocity control) for the entire flight envelope in a disturbed environment.

- numerical results of different flight cases to numerically validate the performance and the interaction between each component of the control architecture.

- disturbance rejection analysis in a critical flight phase under strong wind gusts.

The remainder of this paper is organized as follows. Section II contains the mathematical hybrid UAV model and explains the main features of this UAV class. In Section III, we present the new control architecture based on model-free control algorithms. Subsequently, in section IV, the control performance is analyzed for different flight cases, i.e. hover, transition and forward flight. Finally, section V concludes the paper and introduces the main challenges ahead to improve our control system.

\section{HYBRID UAV MODEL}

This section is subdivided into two parts. First, we present the mathematical formulation of aerodynamic forces and moments, and the aerodynamic assumptions used in the hybrid UAV model. Then, the equations of motion, based in the Newton's second law, are introduced to describe the hybrid UAV behavior.

\section{A. Formulation of aerodynamic forces and moments}

We present an analytic continuous singularity-free formulation of aerodynamic forces $\boldsymbol{F}_{\boldsymbol{a}_{\boldsymbol{b}}} \in \mathbb{R}^{3}$ and moments $M_{a_{b}} \in \mathbb{R}^{3}$ acting in a wing over a complete $360^{\circ}$ angle of attack, based on previous work proposed by [17]. The wing with a surface $S$, is immersed in an incompressible and inviscid airflow with air density $\rho$. The free-stream velocity is composed by the linear element $\boldsymbol{v}_{\infty} \in \mathbb{R}^{3}$ and the angular component defined by $\omega_{\infty} \in \mathbb{R}^{3}$ which, in the absence of wind, is equal to the hybrid UAV angular velocity $\omega_{b} \in \mathbb{R}^{3}$. This formulation of aerodynamic forces and moments is given by :

$$
\left(\begin{array}{c}
\boldsymbol{F}_{\boldsymbol{a}_{\boldsymbol{b}}} \\
\boldsymbol{M}_{\boldsymbol{a}_{\boldsymbol{b}}}
\end{array}\right)=-\frac{1}{2} \rho S \eta C \Phi\left(\boldsymbol{\eta}_{\boldsymbol{b}}\right) C \boldsymbol{\eta}_{\boldsymbol{b}}
$$

where

$$
\eta=\sqrt{v_{\infty}^{2}+\mu c^{2} \omega_{\infty}^{2}}, \text { with } \mu \in \mathbb{R}>0
$$

and

$$
\boldsymbol{\eta}_{\boldsymbol{b}}=\left(\begin{array}{c}
\boldsymbol{v}_{\infty} \\
\boldsymbol{\omega}_{\infty}
\end{array}\right)
$$

The vector $\eta_{b}$ describes the linear and angular free-stream velocities in the body coordinate frame. The matrix $C$ denotes the reference wing parameters in an extended representation,

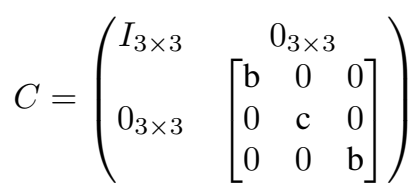

where $b$ and $c$ are, respectively, the wingspan and the mean chord. Finally, the matrix $\Phi \in \mathbb{R}^{6 \times 6}$, which is subdivided into four matrices $\Phi^{(\cdot)} \in \mathbb{R}^{3 \times 3}$, shows the interaction between aerodynamic forces and moments with linear and angular free-stream velocities :

$$
\Phi=\left(\begin{array}{ll}
\Phi^{(f v)} & \Phi^{(f w)} \\
\Phi^{(m v)} & \Phi^{(m w)}
\end{array}\right)
$$

The parameters of $\Phi$ are deduced from thin airfoil theory, we refer the interested reader to [17] for further information. 
Nonetheless, we mention that,

$$
\begin{aligned}
\Phi_{0}^{(f v)} & =\left(\begin{array}{ccc}
C_{d 0} & 0 & 0 \\
0 & C_{y 0} & 0 \\
0 & 0 & 2 \pi+C_{d 0}
\end{array}\right) \\
\Phi^{(f \omega)} & =\left(\begin{array}{ccc}
0 & 0 & 0 \\
0 & 0 & b^{-1} \Delta r C_{y 0} \\
0 & -c^{-1} \Delta r\left(2 \pi+C_{d 0}\right) & 0
\end{array}\right) \\
\Phi_{0}^{(m v)} & =\left(\begin{array}{ccc}
0 & 0 & 0 \\
0 & 0 & -c^{-1} \Delta r\left(2 \pi+C_{d 0}\right) \\
0 & b^{-1} \Delta r C_{y 0} & 0
\end{array}\right) \\
\Phi^{(m \omega)} & =\frac{1}{2}\left(\begin{array}{ccc}
C_{l_{p}} & C_{l_{q}} & C_{l_{r}} \\
C_{m_{p}} & C_{m_{q}} & C_{m_{r}} \\
C_{n_{p}} & C_{n_{q}} & C_{n_{r}}
\end{array}\right)
\end{aligned}
$$

with $C_{d 0}$ the minimal drag coefficient, $C_{y 0}$ the minimal side force coefficient, $\Delta r$ represents the distance between the aerodynamic center and the UAV center of gravity, and $C_{l}, C_{m}$ and $C_{n}$ are the aerodynamic moment coefficients which depend on the angular hybrid UAV velocities $(p, q, r)$. Finally, the flap deflection dynamics are modeled as varying cambered airfoils and the aerodynamic forces and moments created by these deflections $\delta_{j}$ are approximated by the following equations :

$$
\begin{gathered}
\Phi^{(f v)}\left(\delta_{j}\right)=\Phi_{0}^{(f v)}\left(I-\left[\boldsymbol{\xi}_{\boldsymbol{f}}\right]_{\times} \delta_{j}\right) \\
\Phi^{(m v)}\left(\delta_{j}\right)=\Phi_{0}^{(m v)}\left(I-\left[\boldsymbol{\xi}_{\boldsymbol{m}}\right]_{\times} \delta_{j}\right)
\end{gathered}
$$

the flap deflection effectiveness is represented by two skewsymmetric matrices, $\left[\boldsymbol{\xi}_{\boldsymbol{f}}\right]_{\times}$for the force effectiveness and $\left[\boldsymbol{\xi}_{m}\right]_{\times}$for the moment effectiveness, given by :

$$
\begin{gathered}
{\left[\boldsymbol{\xi}_{\boldsymbol{f}}\right]_{\times}=\left[\begin{array}{ccc}
0 & -\xi_{f} & \xi_{f} \\
\xi_{f} & 0 & -\xi_{f} \\
-\xi_{f} & \xi_{f} & 0
\end{array}\right]} \\
{\left[\boldsymbol{\xi}_{\boldsymbol{m}}\right]_{\times}=\left[\begin{array}{ccc}
0 & -\xi_{m} & \xi_{m} \\
\xi_{m} & 0 & -\xi_{m} \\
-\xi_{m} & \xi_{m} & 0
\end{array}\right]}
\end{gathered}
$$

\section{B. Equations of motion}

The hybrid UAV model is divided into four rigid bodies (two propellers and one fuselage composed by two wings) with constant mass $(m)$, represented by ten states $\boldsymbol{x}=\left(\boldsymbol{v}_{\boldsymbol{b}}, \boldsymbol{\omega}_{\boldsymbol{b}}, \boldsymbol{q}\right)$, where $\boldsymbol{v}_{\boldsymbol{b}} \in \mathbb{R}^{3}$ is the vehicle's linear velocity, $\omega_{b} \in \mathbb{R}^{3}$ is the vehicle's angular velocity equals to $\left[\begin{array}{lll}p & q & r\end{array}\right]^{T}$ both expressed in the body coordinate frame and $\boldsymbol{q} \in \mathbb{R}^{4}$ is the quaternion formulation. The system is controlled via four control-inputs, $\boldsymbol{u}=\left(\omega_{l}, \omega_{r}, \delta_{l}, \delta_{r}\right)$, respectively, the left and right propeller rotation speeds and the left and right flap deflections, which are represented in the Fig. 2. In order to compute the forces and moments caused by the wing-propeller interaction, we define two segments. Each segment is composed by one wing $j$ and by one propeller $k$. Thus, the sum of aerodynamic forces acting on the wing $j$ with the thrust $\boldsymbol{T}_{k}$ generated by the propeller rotation $\omega_{k}$ and
TABLE I: Dark0 UAV parameters.

\begin{tabular}{llc}
\hline \hline Parameters & Values & SI Units \\
\hline Mass $(m)$ & 0.492 & {$[\mathrm{Kg}]$} \\
Mean Chord $(c)$ & 0.13 & {$[\mathrm{~m}]$} \\
Wingspan $(b)$ & 0.55 & {$[\mathrm{~m}]$} \\
Wing Area $(S)$ & 0.0743 & {$\left[\mathrm{~m}^{2}\right]$} \\
$J_{x x}$ & 0.0070 & {$\left[\mathrm{Kg} \mathrm{m}^{2}\right]$} \\
$J_{y y}$ & 0.0028 & {$\left[\mathrm{Kg} \mathrm{m}^{2}\right]$} \\
$J_{z z}$ & 0.0061 & {$\left[\mathrm{Kg} \mathrm{m}^{2}\right]$} \\
$J_{p}$ & $5.1116 \times 10^{-6}$ & {$\left[\mathrm{Kg} \mathrm{m}^{2}\right]$} \\
$k_{f}$ & $5.13 \times 10^{-6}$ & {$\left[\mathrm{Kg} \mathrm{m}^{2}\right.$} \\
$k_{m}$ & $2.64 \times 10^{-7}$ & {$\left[\mathrm{Kg} \mathrm{m}{ }^{2}\right]$} \\
$C_{d 0}$ & 0.025 & No units \\
$C_{y 0}$ & 0.1 & No units \\
$C_{l_{p}}$ & 0.2792 & No units \\
$C_{l_{q}}$ & 0.0 & No units \\
$C_{l_{r}}$ & 0.1145 & No units \\
$C_{m_{p}}$ & 0.0 & No units \\
$C_{m_{q}}$ & 1.2715 & No units \\
$C_{m_{r}}$ & 0.0 & No units \\
$C_{n_{p}}$ & 0.081 & No units \\
$C_{n_{q}}$ & 0.0 & No units \\
$C_{n_{r}}$ & 0.0039 & No units \\
$p_{p_{x}}$ & 0.065 & $\mathrm{~m}$ \\
$p_{p_{y}}$ & 0.155 & $\mathrm{~m}$ \\
$p_{p_{z}}$ & 0.0 & $\mathrm{~m}$ \\
$p_{a_{x}}$ & 0.0 & $\mathrm{~m}$ \\
$p_{a_{y}}$ & 0.155 & $\mathrm{~m}$ \\
$p_{a_{z}}$ & 0.0 & $\mathrm{~m}$ \\
$\xi_{f}$ & 0.85 & No units \\
$\xi_{m}$ & 0.55 & No units \\
\hline \hline & & \\
\hline & &
\end{tabular}

the total moment described in the body coordinate frame, are given by :

$$
\begin{aligned}
\boldsymbol{F}_{\boldsymbol{b}} & =\sum_{j, k=1}^{2}\left(\boldsymbol{F}_{\boldsymbol{a}_{b_{j}}}+\boldsymbol{T}_{k}\right) \\
\boldsymbol{M}_{\boldsymbol{b}} & =\sum_{j, k=1}^{2}\left(\boldsymbol{M}_{\boldsymbol{a}_{\boldsymbol{b}_{j}}}+\boldsymbol{\tau}_{\boldsymbol{b}_{\boldsymbol{k}}}+\boldsymbol{p}_{\boldsymbol{p}} \times \boldsymbol{T}_{k}+\boldsymbol{p}_{\boldsymbol{a}} \times \boldsymbol{F}_{\boldsymbol{a}_{\boldsymbol{b}_{j}}}\right)
\end{aligned}
$$

The vector $\boldsymbol{p}_{\boldsymbol{p}}=\left[p_{p_{x}} p_{p_{y}} p_{p_{z}}\right]^{T}$ defines the distance between the propeller $k$ with the hybrid UAV center of mass. Both propellers are positioned symmetrically with respect to the hybrid UAV center of mass. The distance between the aerodynamic center and the center of mass is represented by the vector $\boldsymbol{p}_{\boldsymbol{a}}=\left[\begin{array}{lll}p_{a_{x}} & p_{a_{y}} & p_{a_{z}}\end{array}\right]^{T}$. The internal torque of the propeller $\boldsymbol{\tau}_{\boldsymbol{b}_{\boldsymbol{k}}}$ and its thrust force $\boldsymbol{T}_{k}$, are defined by :

$$
\begin{gathered}
\boldsymbol{T}_{k}=k_{f} \omega_{k}^{2} \overrightarrow{\boldsymbol{x}_{\boldsymbol{b}}}, \quad k_{f} \in \mathbb{R}>0 \\
\boldsymbol{\tau}_{\boldsymbol{b}_{\boldsymbol{k}}}=\boldsymbol{N}_{\boldsymbol{b}_{\boldsymbol{k}}}-J_{p}\left(p+\omega_{j}\right)\left(\begin{array}{c}
0 \\
r \\
-q
\end{array}\right)
\end{gathered}
$$

where

$$
\boldsymbol{N}_{\boldsymbol{b}_{\boldsymbol{k}}}=-\operatorname{sign}\left(\omega_{k}\right) k_{m} \omega_{k}^{2} \overrightarrow{\boldsymbol{x} \boldsymbol{b}}, \quad k_{m} \in \mathbb{R}>0
$$

with $k_{f}$ and $k_{m}$ the propeller force and moment coefficients and $N_{b_{k}}$ is the propeller moment. Equation (15) describes 
the gyroscopic interaction between the propellers and the fuselage with $J_{p}$ equals to the propeller inertia. The vehicle's equations of motion are given by (17) described in the inertial coordinate frame.

$$
\begin{cases}m \dot{\boldsymbol{v}} & =R^{T} \boldsymbol{F}_{\boldsymbol{b}}(\boldsymbol{x}, \boldsymbol{u}, \boldsymbol{W})+m \boldsymbol{g} \\ J \dot{\boldsymbol{\omega}}_{\boldsymbol{b}} & =\boldsymbol{M}_{\boldsymbol{b}}(\boldsymbol{x}, \boldsymbol{u}, \boldsymbol{W})-\left[\boldsymbol{\omega}_{\boldsymbol{b}}\right]_{\times} J \boldsymbol{\omega}_{\boldsymbol{b}} \\ \dot{\boldsymbol{q}} & =\frac{1}{2} \boldsymbol{q} * \boldsymbol{\omega}_{\boldsymbol{b}} \\ \dot{\boldsymbol{p}} & =\boldsymbol{v}\end{cases}
$$

The gravitational acceleration vector is equals to $\boldsymbol{g}=g \overrightarrow{\boldsymbol{z}_{\boldsymbol{i}}}$ and $W \in \mathbb{R}^{3}$ is the wind disturbance vector. The rotation matrix $R$, namely the Direction Cosines Matrix (DCM), represents the UAV rotation in three dimensions as a mathematical formulation. We assume that the hybrid UAV inertia matrix $J$ is diagonal and it equals to $J=\operatorname{diag}\left[J_{x x} J_{y y} J_{z z}\right]$. The position vector in the inertial coordinate frame is represented by $\boldsymbol{p}=\left[\begin{array}{lll}x & y & z\end{array}\right]^{T}$. The highly maneuverable nature of the vehicle calls for a global numerically stable formulation of attitude and justifies the use of quaternions. The symbol $*$ in the previous equation corresponds to the quaternion product. All hybrid UAV parameters used in this paper correspond to the Darko UAV shown in Fig. 2, and they are described in the Table I.

\section{CONTROL STRATEGY}

The proposed control algorithm has no information about the Hybrid UAV parameters (e.g. mass, inertia, aerodynamic coefficients, etc.). Though, we use a prior knowledge of signconvention between control-input influence in the UAV states based on flight mechanics equations to develop the correct interaction of blocks in the control architecture. Therefore, the model given in the previous section is used only to simulate the hybrid UAV system and not for control design.

\section{A. Model-Free Control Principles}

As introduced by [18], an unknown finite-dimensional system with a single control-input $(u)$ and a single output (y) can be described by the following input/output relation in a differential equation formulation :

$$
\mathbb{E}\left(y, \dot{y}, \ldots, y^{(a)}, u, \dot{u}, \ldots, u^{(b)}\right)=0
$$

where $\mathbb{E}$ is a polynomial function with real unknown coefficients. We can also describe

$$
y^{v}=\mathbb{E}\left(t, y, \dot{y}, \ldots, y^{(v-1)}, y^{(v+1)}, \ldots, y^{(a)}, u, \dot{u}, \ldots, u^{(b)}\right)
$$

with $0<v \leq a$ and $\frac{\delta \mathbb{E}}{\delta y^{v}} \neq 0$. This unknown dynamic can be modeled by a purely numerical equation, so-called UltraLocal Model :

$$
y_{m}^{(v)}=F_{y}+\lambda \cdot u
$$

In (20), $v$ is the order derivative of $y_{m}, \lambda \in \mathbb{R}$ is a non-physical constant parameter. Moreover, the exploitation of this numerical model requires the knowledge of $F_{y}$. This quantity represents the real dynamics of the model as well as the different disturbances which could damage the performance of the output-system. Thus, an accurate

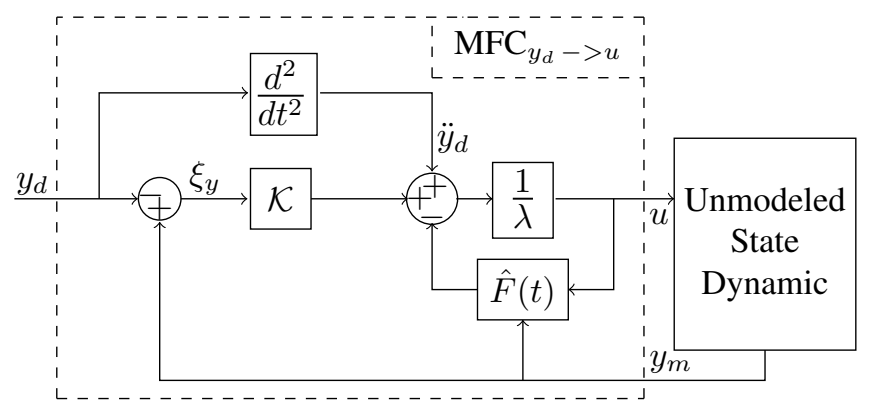

Fig. 3: Overview of Model-Free Control schema.

estimation of $F$, defined as $\hat{F}$, is crucial and plays an import role in the control performance. Assuming that we do not have any information about the plant, its estimation can be computed directly by the following methodology in which we use a second-order Ultra-Local Model :

$$
\ddot{y}_{m}=F_{y}+\lambda \cdot u
$$

The first step is to apply the Lapace Transform in (21). Referring to elementary operational calculus we transform (21) to $(22)$ :

$$
s^{2} Y_{m}(s)-s y_{m}(0)-\dot{y}_{m}(0)=\frac{F_{y}}{s}+\lambda U(s)
$$

Where $Y_{m}(s)$ and $U(s)$ correspond to the Laplace transforms of $y_{m}$ and $u$. By differentiating twice the previous equation we are able to rid the initial conditions $y_{m}(0)$ and $\dot{y}_{m}(0)$ :

$$
2 Y_{m}(s)+4 s \frac{d Y_{m}(s)}{d s}+s^{2} \frac{d^{2} Y(s)}{d s^{2}}=\frac{2 F_{y}}{s^{3}}+\lambda \frac{d^{2} U(s)}{d s^{2}}
$$

However, the variable $s$ in the time domain corresponds to the derivation with respect to time that is sensitive to noise corruptions and can amplify the noise measurement. Therefore, in order to reduce both noise and numerical computation errors on the output estimation, we replace the derivative terms by integrators $\left(\frac{1}{s}\right)$ who have robust properties with respect to noise. Thus, multiplying both sides of (23) by $s^{-3}$, we obtain :

$$
\frac{2 Y_{m}(s)}{s^{3}}+\frac{4}{s^{2}} \frac{d Y_{m}(s)}{d s}+\frac{1}{s} \frac{d^{2} Y(s)}{d s^{2}}=\frac{2 F_{y}}{s^{6}}+\frac{\lambda}{s^{3}} \frac{d^{2} U(s)}{d s^{2}}
$$

Equation (24) can be transferred back to the time domain employing elementary calculus and Cauchy's formula to reduce multiple integrals in a simple one :

$$
\begin{gathered}
\hat{F}_{y}=\frac{5 !}{2 T^{5}} \int_{t-T}^{t}\left[(T-\sigma)^{2}-4 \sigma(T-\sigma)+\sigma^{2}\right] y_{m}(\sigma) \\
-\left[\frac{\lambda}{2} \sigma^{2}(T-\sigma)^{2} u(\sigma)\right] d \sigma
\end{gathered}
$$

From measurements of $y_{m}$ and $u$ obtained in the last $T$ seconds, the unmodeled dynamic of $y$ and the disturbances are estimated by $\hat{F}_{y}$ which is updated for each interval of integration $[t-T, t]$. This interval corresponds to the window width of a receding horizon strategy which results in a tradeoff. The idea is to choose the window width small so as to calculate the estimation within an acceptable short delay 


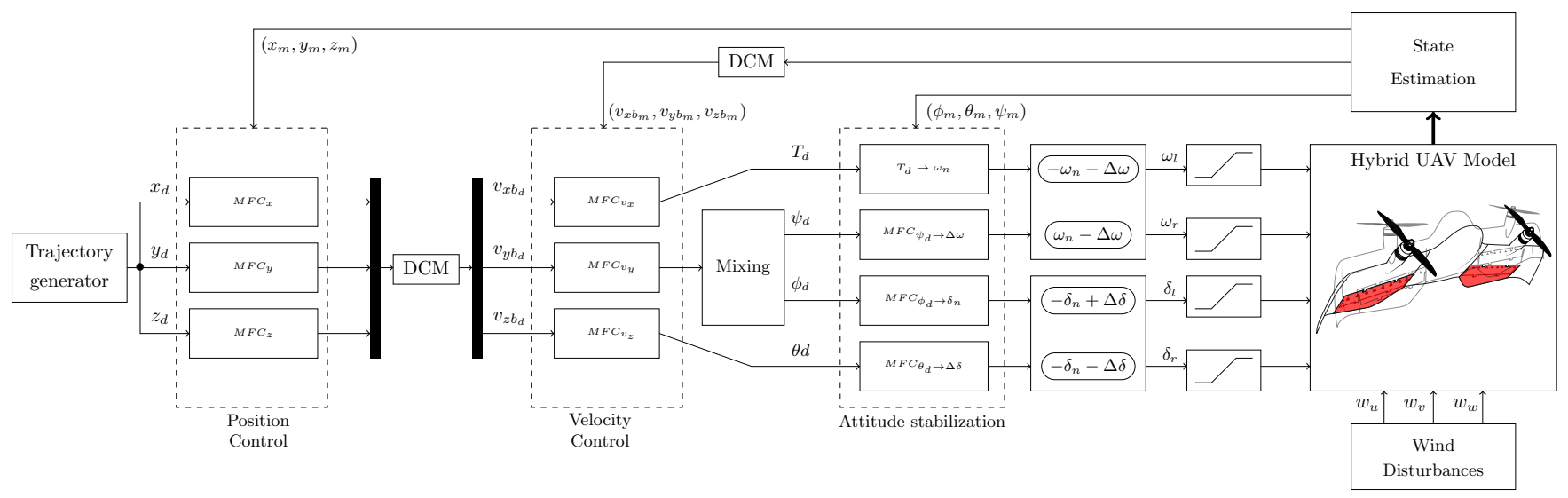

Fig. 4: Cascaded MFC architecture designed for hybrid Unmanned Aerial Vehicles. Position control blocks send desired velocities for the velocity control blocks that compute the necessary thrust value as well as the references for attitude stabilization control loop. Based on these desired values, propeller speeds $\left(\omega_{l}, \omega_{r}\right)$ and flap deflections $\left(\delta_{l}, \delta_{r}\right)$ are defined.

but large enough in order to preserve the low-pass filter properties whose noise attenuations of $y_{m}$. Based on such estimator, it is possible to design a robust controller that estimates the system dynamic on-line by a piecewise constant function $\hat{F}_{y}$ periodically updated for each measure of $y_{m}$ and $u$. The general form of the control-input can be defined as in the Fig. 3 and is given by,

$$
u=\underbrace{-\frac{\hat{F}_{y}}{\lambda}}_{\text {Nonlinear Cancellation }}+\underbrace{\frac{y_{d}^{(2)}+\mathcal{K}(\xi)}{\lambda}}_{\text {Closed loop tracking }}
$$

where the quantity $\xi=y_{m}-y_{d}$ is the tracking error and $\mathcal{K}(\xi)$ is a closed loop feedback controller, usually defined as a proportional, proportional-derivative or even so as proportional-integral-derivative gain. In this paper, we define the closed loop feedback controller as a proportional $K_{p}$ and derivative gain $K_{d}$. We recognize in (25) the typical mathematical expression of a nominal control in the flatnessbased in which the non-linear terms $\hat{F}_{y}$ is added with a closed loop tracking of a reference trajectory $t \rightarrow y_{d}(t)$. The error dynamic can be deduced from the combination of (25) with (21) :

$$
\ddot{\xi}_{y}=\ddot{y}_{m}-\ddot{y}_{d}=\overbrace{F_{y}-\hat{F}_{y}}^{\xi_{F} \approx 0}+K_{p} \xi_{\theta}+K_{d} \dot{\xi}_{\theta}
$$

Note that, if the error $\left(\xi_{F_{y}}\right)$ between the estimator and the true dynamic, is approximately zero during $[t-T, t]$, a simple proportional-derivative controller will be enough to ensure the error convergence to zero. Whereas, an integration effect is implicitly involved in the model-free control algorithm.

\section{B. Control architecture}

Figure 4 shows the main ideas of our control architecture. The block Trajectory generator is composed of a state flow algorithm that defines constantly the desired positions $\left(x_{d}, y_{d}\right.$ and $\left.z_{d}\right)$ in the inertial coordinate system. These references are taken into account by the Position control block and are compared with the respective measures $\left(x_{m}\right.$, $\left.y_{m}, z_{m}\right)$ creating three errors that are minimized by the MFC algorithms in the Position control block. These three MFC algorithms in charge of the position tracking, also compute the desired velocity in their respective axes. These references values which are defined in the inertial coordinate frame are transformed to the body coordinate frame as well as the velocities measurements. Thus, the velocity control $\mathrm{MFC}_{v_{x}}$ computes the required thrust $T_{d}$ to reach this desired velocity along $\overrightarrow{\boldsymbol{x}_{\boldsymbol{b}}}$, the block $\mathrm{MFC}_{v_{z}}$ assures the velocity control along $\overrightarrow{z_{b}}$ and determine the necessary pitch angle $\theta_{d}$ to reach this desired velocity $v_{b_{z_{d}}}$. Both blocks control their respective velocities and inform the desired thrust and pitch angle for the entire flight envelope, i.e. hover, transition and forward flight. However, the velocity control along $\overrightarrow{\boldsymbol{y}_{\boldsymbol{b}}}$ is designed depending on the current hybrid UAV flight phase. Therefore, in hover flight, the block $\mathrm{MFC}_{v_{y}}$ set the desired yaw angle $\psi_{d}$ and the block $\mathrm{MFC}_{\psi_{d}}$ actuates in the system by a differentialthrust command creating a moment around $\overrightarrow{z_{b}}$ in order to reach the desired velocity along $\overrightarrow{\boldsymbol{y}_{\boldsymbol{b}}}$. In forward flight, this lateral velocity is reached from roll rotations around $\overrightarrow{\boldsymbol{x}_{\boldsymbol{b}}}$. These rotations orient the lift force and the hybrid UAV can perform left-right turns with, respectively, negative and positive roll angles $\phi$. The propeller speeds $\left(\omega_{l}, \omega_{r}\right)$ are defined by the sum of nominal propeller rotation $\omega_{n}$ with a differential propeller speed $\Delta_{\omega}$ which is in charge of the yaw control. The negative sign of $\omega_{n}$ for the left-propeller $\omega_{l}$ is due to the counter-rotation sense. And the flap-deflections $\left(\delta_{l}, \delta_{r}\right)$, which are in convention negative for pitch-up, are composed by the sum of symmetrical flap deflection $\delta_{n}$ with anti-symmetrical flap deflections $\Delta_{\delta}$ that are respectively the control-input for the pitch angle $\theta$ and for the roll angle $\phi$.

\section{SIMULATION RESULTS}

In order to investigate the performance of the modelfree control architecture, we design a set of numerical flight simulations with distinct target scenarios that include vertical take-off and landing, hover-to-forward transition, forwardto-hover transition and forward flights. All simulations are 


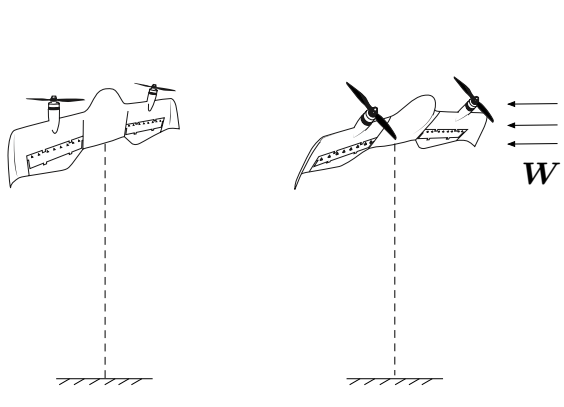

(a)

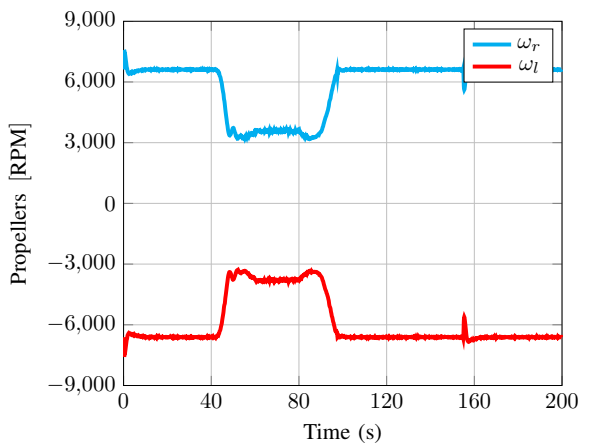

(d)

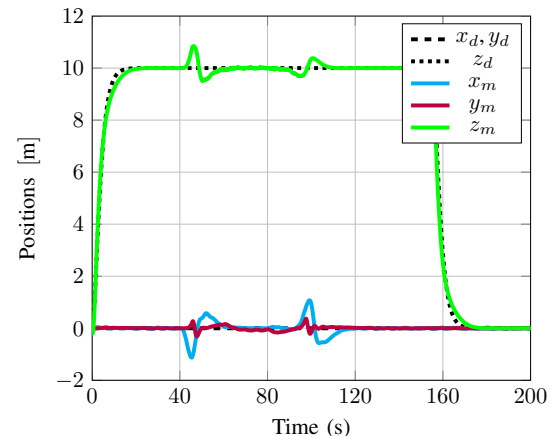

(b)

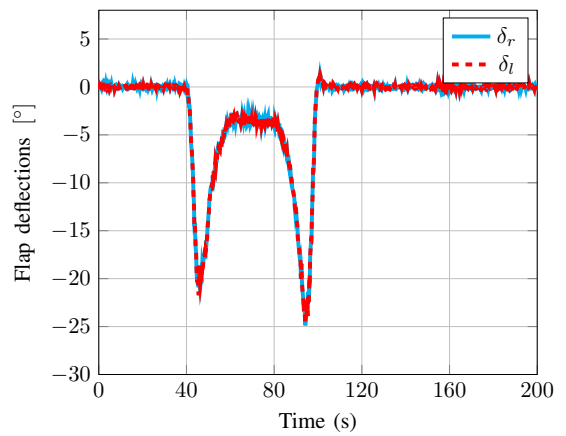

(e)

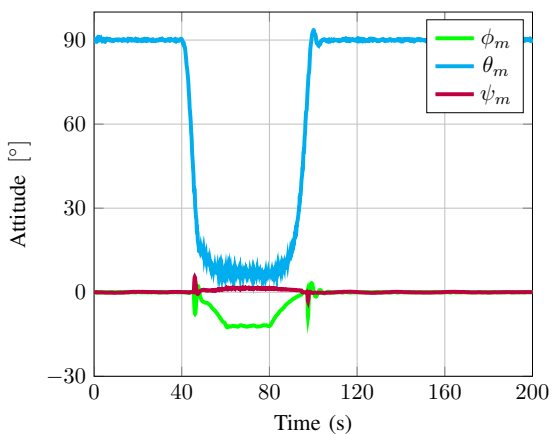

(c)

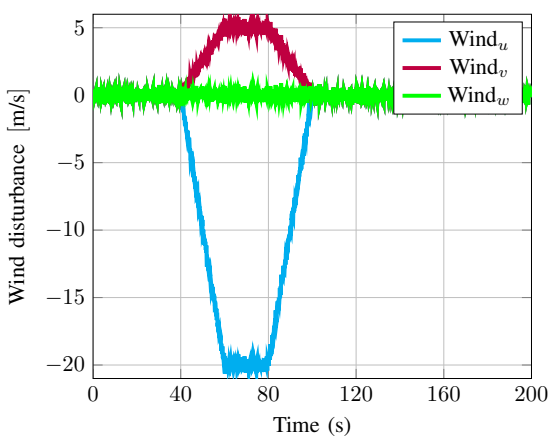

(f)

Fig. 5: (\#Flight 1) - Vertical take-off and transition flight to assure position tracking during high wind disturbances. On the top, from left to right: simulation illustration, positions in the inertial coordinate frame and attitude. On the bottom: propeller speeds $\left(\omega_{l}<0\right.$ and $\left.\omega_{r}>0\right)$ due to counter-rotation sense, flap deflections $\left(\delta_{l}\right.$ and $\left.\delta_{r}\right)$ convention negative for pitch-up, and wind disturbance.

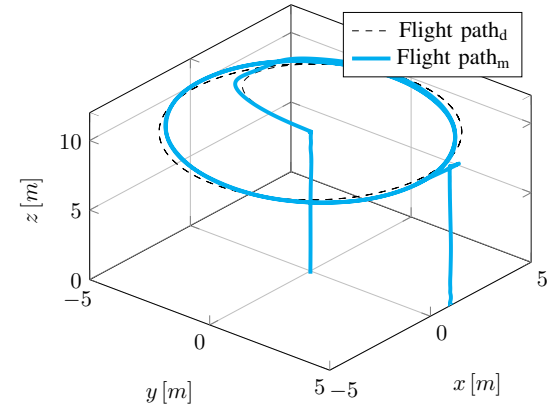

(a)

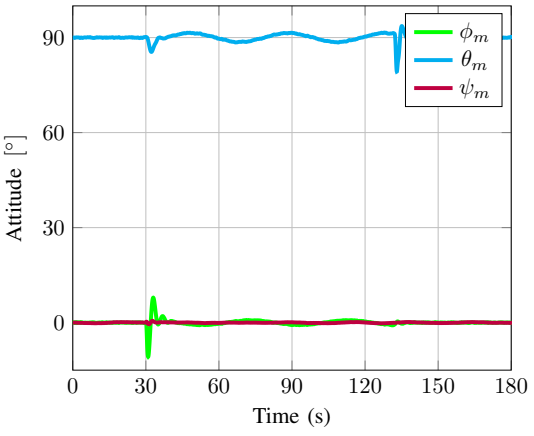

(d)

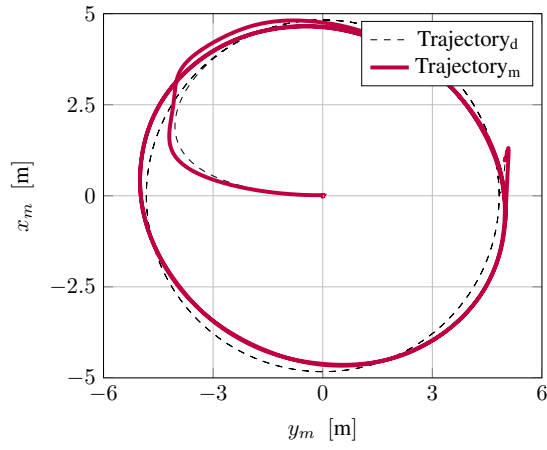

(b)

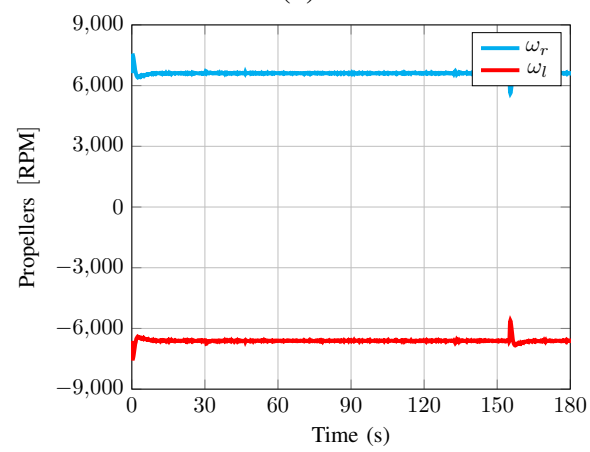

(e)

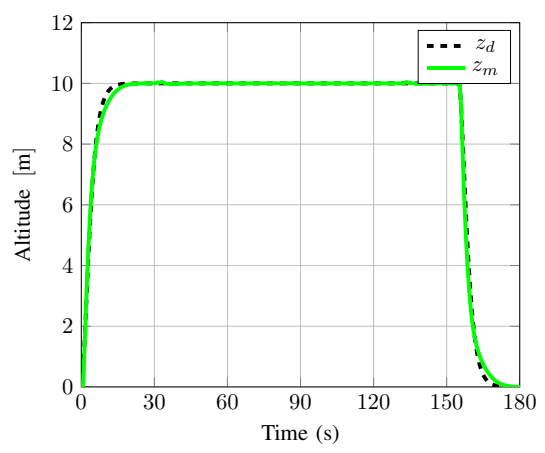

(c)

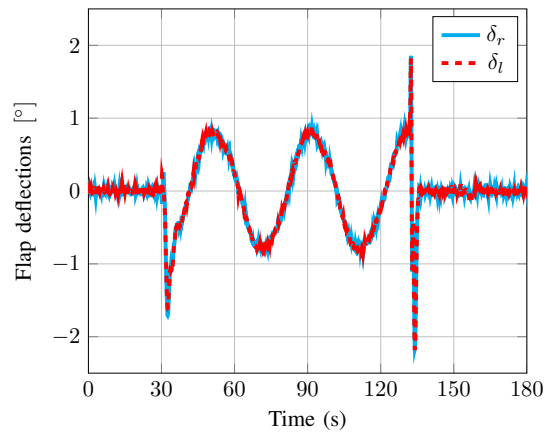

(f)

Fig. 6: (\#Flight 2) - Circular position tracking in hover flight mode. On the top, from left to right: the 3D flight path, North and East positions and altitude. On the bottom: attitude, propeller speeds and flap deflections. 


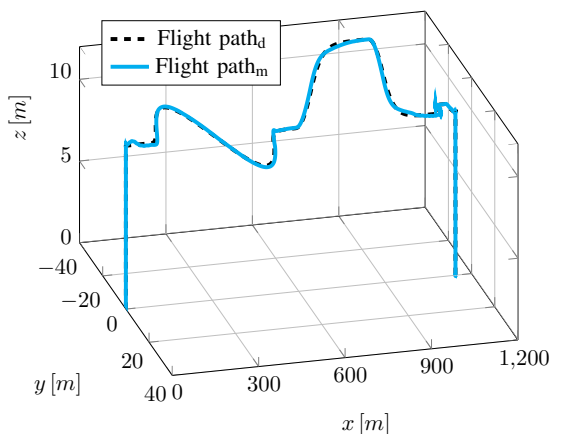

(a)

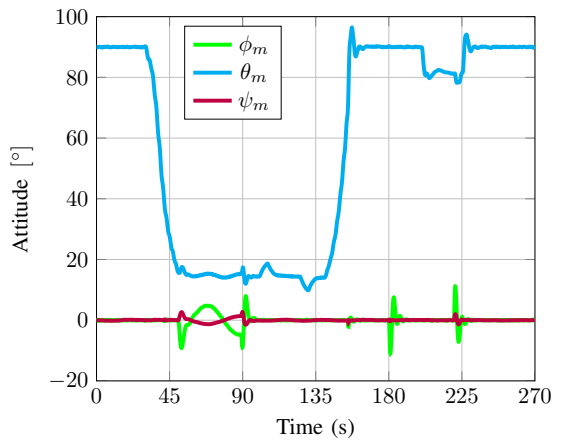

(d)

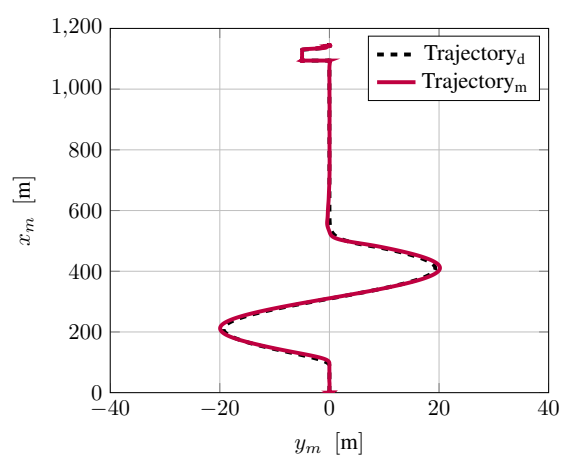

(b)

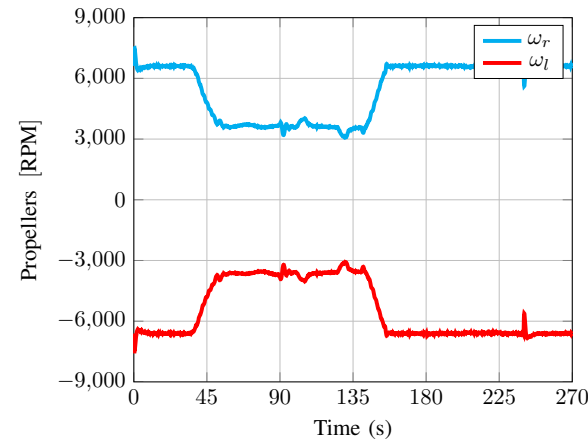

(e)

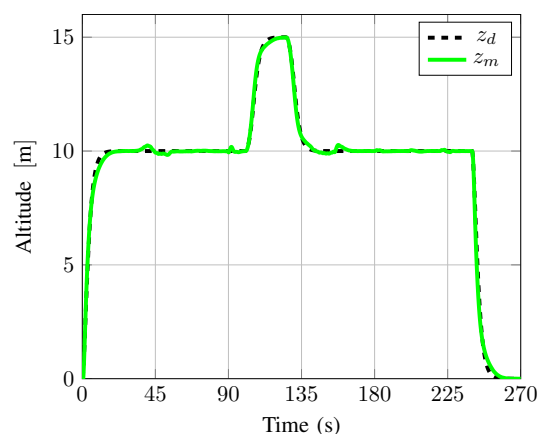

(c)

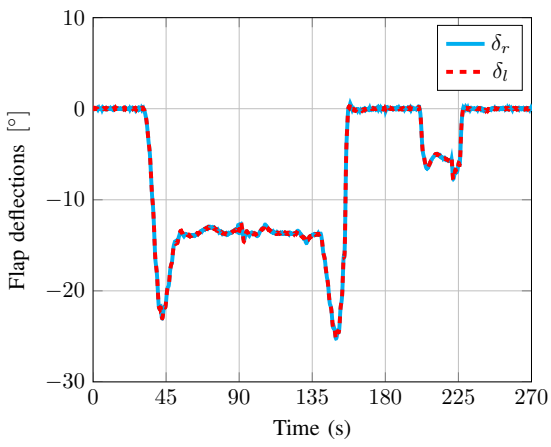

(f)

Fig. 7: (\#Flight 3) - Entire flight envelope simulation in relatively calm flight conditions. On the top, from left to right: the 3D flight path, North and East positions and altitude. On the bottom: attitude, propeller speeds and flap deflections.

discretized at $500 \mathrm{~Hz}$ and include noise measurements and wind disturbances. An invariant observer [19] is used to estimate the UAV states providing a smoother signal, this operation adds delays in the closed loop and must be taken into account during the controller's synthesis. The MFC parameters were tuned for the entire flight envelope and are the same for all simulations.

\section{A. Flight simulations}

In hover flight mode, we analyzed two flight tests. The main objective of the first flight simulation shown in the Fig. 5, is the study of wind influence in the position tracking (\#Flight 1), for the following desired positions :

$$
\begin{aligned}
& x_{d}=0, \forall t \\
& y_{d}=0, \forall t \\
& z_{d}= \begin{cases}10, & t \in[0 ; 155] s \\
0, & t>155 s\end{cases}
\end{aligned}
$$

Indeed, during this flight mode the hybrid UAV is more susceptible to aerodynamic disturbances. We can explain this by the fact that, in the vertical position, the wind gust along the $z_{b}-$ axis is in contact with the total reference wing area generating a considerable drag force. Also, the hybrid UAV is not able to compensate this force in the vertical position. Which is why, the transition is performed and the drag force created by the wind is now along the $x_{b}-$ axis. This makes the vehicle add thrust in order to compensated this undesirable aerodynamic force in order to assure the position tracking. The thrust used to reject this perturbation can be seen in the Fig. 5d. And the wind from east with a magnitude of $5 \mathrm{~m} / \mathrm{s}$, see Fig. $5 \mathrm{f}$, also produces a drag force in the $y_{b}$-axis. This force is compensated by orienting the lift force with a symmetrical rotation around the $x_{b}-$ axis, the roll angle shown in the Fig. 5c. In the second flight simulation, we impose a circular desired path (\#Flight 2) in order to validate the interaction between all control blocks in the proposed control architecture. The following equations define the desired trajectories $\left(x_{d}, y_{d}, z_{d}\right)$,

$$
\begin{aligned}
& x_{d}= \begin{cases}0, & t<30 s \\
x_{c}+r \cos \left(\frac{2 \pi}{40} t\right), & t \in[30 ; 130] s \\
1, & t>130 s\end{cases} \\
& y_{d}= \begin{cases}0, & t<30 s \\
y_{c}+r \sin \left(\frac{2 \pi}{40} t\right), & t \in[30 ; 130] s \\
5, & t>130 s\end{cases} \\
& z_{d}= \begin{cases}10, & t \in[0 ; 155] s \\
0, & t>155 s\end{cases}
\end{aligned}
$$

where $x_{c}$ and $y_{c}$ correspond to the center of the circle and $r$ is its radius. This maneuver requires that the hybrid UAV follow a circular trajectory while stabilizing its attitude. Accurate position, velocity and attitude control are needed to accurately follow the desired flight plan with the desired attitude orientation. Figure 6 shows the simulation results. 
Finally, a complete flight mission (\#Flight 3) is presented in the Fig. 7 in which we evaluate all hybrid UAV flight capabilities through a vertical take-off from zero to ten meters of altitude followed by the transition to forward flight with a position tracking in the $x y$ - plane and an altitude change in forward flight. Then, the forward-to-hover transition is performed and the simulation ends with a vertical landing. The complete 3D flight path is presented in the Fig. 7a. The controller assures the position tracking during the entire mission, as we can see in the Fig. 7b. The altitude presents small oscillations at 40 and 140 seconds of simulation which is quite acceptable for this UAV class. These oscillations are due to the fast variations of aerodynamic forces and moments that occur during the transition flight phase where the pitch angle decreases causing an important variation in the angle of attack, see Fig. 7d.

\section{B. Results analysis}

The controller's performance was evaluated through a quantitative analysis for all previous flight simulations. So, in this analysis, we compute the root mean square error for the vertical take-off and transition flight under high wind disturbances (\#Flight 1), circular position tracking in hover (\#Flight 2) and for the entire flight envelope (\#Flight 3). The results are presented in the Table II, with a RMSE less than 0.8 meter, 0.6 meter per second and 0.8 degrees, respectively, for the position tracking, velocity control and for the attitude stabilization.

TABLE II: Root Mean Square Errors - RMSE

\begin{tabular}{lrrrc}
\hline \hline States & \#Flight 1 & \#Flight 2 & \#Flight 3 & SI Units \\
\hline$x$ & 0.2335 & 0.2348 & 0.7140 & {$[\mathrm{~m}]$} \\
$y$ & 0.0636 & 0.1258 & 0.3681 & {$[\mathrm{~m}]$} \\
$z$ & 0.1913 & 0.1384 & 0.1335 & {$[\mathrm{~m}]$} \\
$v_{x b}$ & 0.1505 & 0.0897 & 0.1052 & {$[\mathrm{~m} / \mathrm{s}]$} \\
$v_{y b}$ & 0.0849 & 0.0899 & 0.0612 & {$[\mathrm{~m} / \mathrm{s}]$} \\
$v_{z b}$ & 0.5523 & 0.1135 & 0.3684 & {$[\mathrm{~m} / \mathrm{s}]$} \\
$\phi$ & 0.1968 & 0.0183 & 0.1464 & {$\left[^{\circ}\right]$} \\
$\theta$ & 0.7720 & 0.1800 & 0.6094 & {$\left[^{\circ}\right]$} \\
$\psi$ & 0.1434 & 0.1553 & 0.0416 & {$\left[^{\circ}\right]$} \\
\hline \hline
\end{tabular}

\section{CONCLUSIONS}

In this paper, we focused on the development of a modelfree control architecture of hybrid UAVs. The proposed controller was designed to stabilize the entire flight envelope of hybrid UAVs including vertical take-off and landing, transition and forward flight with no information about its parameters. Simulation results shown an effective control performance for the entire flight envelope and excellent disturbance rejections during the critical flight phases. Furthermore, the control strategy overcomes the tightly nonlinear, coupled and under-actuated nature of the hybrid UAV. Also, takes into account the many degrees of freedom of the system and ensures attitude stability, velocity control and position tracking for all flight phases.

\section{REFERENCES}

[1] K. Alexis, A. Tzes, and G. Nikolakopoulos, "Model predictive quadrotor control: attitude, altitude and position experimental studies," IET Control Theory \& Applications, Vol. 6, No. 12, pp. 1812-1827, March 2012.

[2] T. Espinoza-Fraire, A. Dzul, F. Cortés-Martínez, and W. Giernack, "Real-time Implementation and Flight Tests using Linear and Nonlinear Controllers for a Fixed-wing Miniature Aerial Vehicle (MAV)," International Journal of Control, Automation and Systems, Vol. 16, No. 1, pp. 392-396, February 2018.

[3] R. Hugh Stone, P. Anderson, C. Hutchison, A. Tsai, P. Gibbens, and K. C. Wong, "Flight Testing of the T-Wing Tail-Sitter Unmanned Air Vehicle," Journal of Aircraft, Vol. 45, No. 2, pp. 673-685, April 2008.

[4] N. B. Knoebel, and T. W. McLain, "Adaptive Quaternion Control of a Miniature Tailsitter UAV," American Control Conference, Seattle, Washington, USA, pp. 2340-2345, June 2008.

[5] J. Hauser, S. Sastry, and G. Meyer, "Nonlinear control design for slightly non-minimum phase systems: application to v/stol aircraft," Automatica, Vol. 28, pp. 665-679, January 1992.

[6] D. Pucci, T. Hamel, P. Morin, and C. Samson, "Nonlinear Control of Aerial Vehicles Subjected to Aerodynamic Forces," IEEE Conference on Decision and Control (CDC), Florence, Italy, pp. 4839-4846, December 2013.

[7] G. R. Flores-Colunga, and R. Lozano-Leal, "A Nonlinear Control Law for Hover to Level Flight for the Quad Tilt-rotor UAV," Proceedings of the 19th World Congress The International Federation of Automatic Control, South Africa, pp. 11055-11059, August 2014.

[8] A. Frank, J.S. McGrew, M. Valenti, D. Levine, and J.P. How, "Hover, transition, and level flight control design for a single-propeller indoor airplane." In Proc. AIAA Guidance, Navigation Control Conference, Hilton Head, South Carolina, pp. 1-43, August 2007.

[9] L. R. Lustosa, F. Defay, and J.-M. Moschetta, "Longitudinal study of a tilt-body vehicle: modeling, control and stability analysis," International Conference on Unmanned Aircraft Systems (ICUAS), Denver, Colorado, USA, pp. 816-824, June 2015.

[10] J. Zhou, L. Ximin, Z. Li, S. Shen, and F. Zhang, "A Unified Control Method for Quadrotor Tail-sitter UAVs in All Flight Modes: Hover, Transition, and Level Flight," International Conference on Intelligent Robots and Systems (IROS), pp. 4835-4841, September 2017.

[11] E.J.J. Smeur, M. Bronz, and G.C.H.E. de Croon, "Incremental control and guidance of hybrid aircraft applied to the Cyclone tailsitter UAV," to be published.

[12] Haoping Wang, Xuefei Ye, Yang Tian, Gang Zheng, and Nicolai Christov, "Model-Free Based Terminal SMC of Quadrotor Attitude and Position," IEEE Transactions on Aerospace and Electronic Systems, Vol. 52, No, 5, pp. 2519-2528, October 2016.

[13] Y. Al Younes, A. Drak, H. Noura, A. Rabhi, and A. El Hajjaji, "Robust Model-Free Control Applied to a Quadrotor UAV," Journal of Intelligent \& Robotic Systems, Vol. 84, No. 1-4, pp. 37-52, December 2016.

[14] J. M. O. Barth, J.-P. Condomines, M. Bronz, L. R. Lustosa, J.-M. Moschetta, C. Join, and M. Fliess, "Fixed-wing UAV with transitioning flight capabilities : Model-Based or Model-Free Control approach? A preliminary study," International Conference on Unmanned Aircraft Systems (ICUAS), Dallas, TX, USA, USA, pp. 1157-1164, June 2018.

[15] J. M. O. Barth, J.-P. Condomines, J.-M. Moschetta, C. Join, and M. Fliess, "Model-Free Control Approach for Fixed-Wing UAVs with Uncertain Parameters Analysis," 23rd International Conference on Methods and Models in Automation and Robotics (MMAR), Miedzyzdroje, Poland, pp. 527-532, August 2018.

[16] O. Bara, M. Fliess, C. Join, J. Daye, and S. M. Djouadi, "Toward a model-free feedback control synthesis for treating acute inflammation," Journal of Theoretical Biology, Elsevier, Vol. 448, No. 7, pp. 26-37, July 2018.

[17] L. R. Lustosa, F. Defay, and J.-M. Moschetta, "Global Singularity-Free Aerodynamic Model for Algorithmic Flight Control of Tail Sitters," AIAA Journal of Guidance, Control, and Dynamics, Vol. 42, No. 2, pp. 303-316, February 2019.

[18] M. Fliess, and C. Join, "Model-free control," International Journal of Control, Taylor \& Francis, Vol. 86, No. 12, pp. 2228-2252, July 2013.

[19] P. Martin, and E. Salaun, "Design and implementation of a lowcost observer-based attitude and heading reference system," Control Engineering Practice, Vol. 18, No. 7, pp. 712-722, July 2010. 